Case Report

\title{
Acute Abdominal Compartment Syndrome following Extraperitoneal Bladder Perforation
}

\begin{abstract}
Ana Licina
Austin Health, 145 Studley Road, Heidelberg, VIC 3084, Australia

Correspondence should be addressed to Ana Licina; analicina@hotmail.com

Received 27 January 2017; Revised 8 April 2017; Accepted 11 May 2017; Published 30 May 2017

Academic Editor: Jian-jun Yang

Copyright (C) 2017 Ana Licina. This is an open access article distributed under the Creative Commons Attribution License, which permits unrestricted use, distribution, and reproduction in any medium, provided the original work is properly cited.

Extraperitoneal bladder perforation is a known complication of a commonly performed rigid cystoscopy. If unrecognized, this complication can lead to continuous intra-abdominal fluid leakage with consequent organ function impairment and symptoms. This is the first case report in literature of a transurethral bladder perforation causing an acute abdominal compartment syndrome, which was subsequently managed conservatively with supportive management only. Case Presentation. We describe a clinical course of a 73-year-old Caucasian female whose initial acute presentation involved urinary symptoms. Surgery and general anaesthesia during rigid cystoscopy were complicated by an initially unrecognized extraperitoneal bladder perforation, resulting in fluid extravasation. This extravasation resulted in transurethral bladder resection syndrome with acute intra-abdominal free fluid accumulation. This complication caused acute abdominal compartment syndrome resulting in respiratory end-organ compromise and immediate postextubation respiratory failure. Patient required an emergency reintubation. During the management, diagnosis was considered through the use of the point of care abdominal ultrasound. Postoperatively, patient was managed conservatively in intensive care. Postoperative course included an approximate nine liters of urinary diuresis and supportive ventilation for four days. Conclusion. There is equipoise in the clinical management of abdominal compartment syndrome with regard to supportive medical management alone or invasive surgical treatment.
\end{abstract}

\section{Introduction}

Asymptomatic bladder perforation during a rigid cystoscopy and transurethral bladder tumour treatment may occur in up to $58 \%$ of patients [1].

If severe, intraoperative bladder perforation can result in intra-abdominal fluid accumulation causing intra-abdominal hypertension (IAH). IAH is defined as a sustained intraabdominal pressure greater than $12 \mathrm{mmHg}$ [2].

If IAH is severe, it can result in abdominal compartment syndrome.

We describe the clinical course of a patient who experienced an unrecognized extraperitoneal bladder perforation, leading to massive intra-abdominal fluid accumulation, IAH, and subsequently ACS.

In this case, acute IAH caused immediate respiratory failure on extubation due to a mass effect of fluid and subsequent lung compression.
Further notable features of this case are the physiological changes related to the perforation of the bladder with a fluid overload pattern and electrolyte derangement distinct from the transurethral prostate syndrome [3]. We note the differences between the two in the Discussion.

\section{Case Description}

This seventy-three-year-old Caucasian female with a prior history of endometrial cancer and suspected recurrence demonstrated on CT abdomen and pelvis was booked for a rigid cystoscopy and urgent bilateral ureteric stent insertion.

This investigation demonstrated a potential localized recurrence of the tumour with bilateral compression of the ureters resulting in bilateral hydronephrosis.

Her past medical history included a hysterectomy, radiotherapy, and chemotherapy for endometrial carcinoma 2 
years ago. Other comorbidities included noninsulin dependent diabetes, hypertension, and body mass index of 39. Renal function was acutely impaired with $\mathrm{Cr}=200 \mu \mathrm{mol} / \mathrm{l}$ and estimated GFR was $30 \mathrm{~mL} / \mathrm{min} / 1.73 \mathrm{~m} 2$.

Uneventful modified RSI was performed with a size 7 standard endotracheal tube for airway management and maintenance. During the course of surgery, anaesthesia was initially uneventful. Approximately thirty minutes from the start of the procedure, there was an unexplained rise in airway pressures from an airway peak pressure of 35 to 40 .

This coincided with the neuromuscular paralysis wearing off and the patient was given a further dose of atracurium. High airway pressures moderated with paralysis and an alternative ventilation strategy.

Bilateral ureteric stents were inserted with real-time radiological guidance and correct positioning was confirmed. Throughout the procedure, ongoing bladder washout was continuing with the irrigation fluid containing glycine $1.5 \%$ with a total volume of ten liters used. The washout solution was hung at standard height of two meters and rate of infusion controlled by the operating surgeon. The procedure was deemed technically difficult and took ninety minutes of surgical time to complete.

Patient was extubated once all extubation-readiness criteria were met. Immediately after the endotracheal tube (ETT) was removed, patient became agitated, diaphoretic, and progressively centrally cyanosed. Haemodynamic instability was noted with severe hypertension with systolic blood pressure reaching $200 \mathrm{mmHg}$.

Decision was made to reintubate the patient.

Once the ETT was resecured uneventfully, the usual algorithm for respiratory distress was followed and definitive diagnosis for severe diaphoresis was not made [4].

Further large bore intravenous access was obtained and $20 \mathrm{~g}$ radial arterial line was inserted. The patient was examined in her entirety and the expanding abdominal girth was noted by the theatre team. Ultrasound fast scan was performed and a significant amount of free fluid was noted surrounding the spleen and the left kidney.

Comparison was made with the CT scan obtained 24 hours prior to the procedure, where no free fluid in the abdomen was seen (Figure 1).

The washout solution at this stage was changed to normal saline and rate was decreased as per surgical team instructions.

First blood gas on 100\% oxygen after reintubation and arterial line insertion demonstrated hyponatraemia: $\mathrm{pO} 2=$ $184 \mathrm{mmHg}, \mathrm{pCO} 2=54 \mathrm{mmHgm}, \mathrm{Na}=126 \mathrm{mmol} / \mathrm{L}, \mathrm{K}=$ $3.8 \mathrm{mmol} / \mathrm{L}$, and $\mathrm{HCO} 3=21 \mathrm{mmol} / \mathrm{L}$.

CT scan chest/abdomen and cystogram confirmed a bladder perforation and intra-abdominal fluid extravasation as illustrated in Figure 2. Continuous bladder irrigation was ceased and patient was taken to intensive care intubated.

Supportive management was continued in intensive care and she remained intubated for further 4 days. Supportive medical management included respiratory system support through artificial ventilation, sedation facilitating ongoing intubation and improving the abdominal wall compliance.

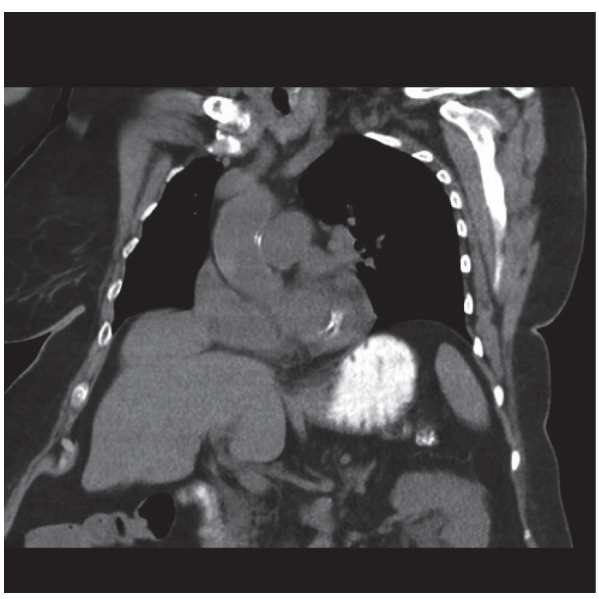

FIgURE 1: Sagittal CT scan demonstrating no free fluid in the abdomen.

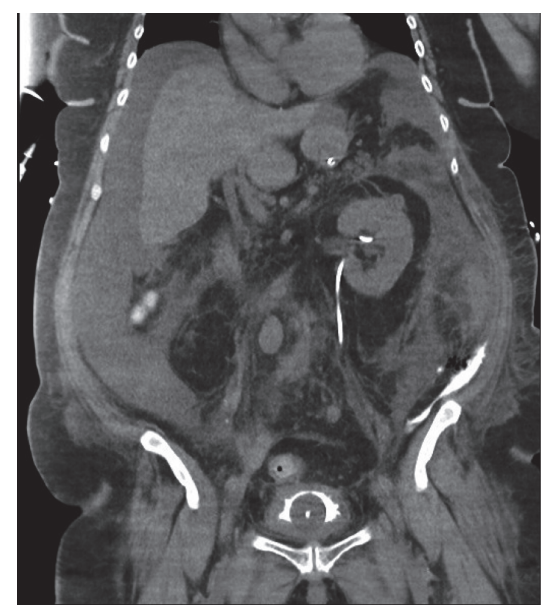

FIGURE 2: Sagittal CT scan illustrating fluid accumulation around the liver and in the R paracolic gutter.

After the initial episode of hypertension at reintubation, there was no further haemodynamic instability.

Diuresis was achieved through use of daily loop diuretics. Electrolytes were carefully monitored. As there were no clinical signs of hyponatraemia, hypertonic saline was not used. There was no abdominal percutaneous tap performed in order to drain the fluid and there was no exploratory laparotomy performed either. As expected, this patient developed pulmonary oedema with clinical evidence on CXR and impaired gas exchange. Eight and a half liters of fluid was diuresed during the intensive care stay. Patient was extubated uneventfully and discharged to the ward.

After an initial persistent hyponatraemia, sodium returned to normal values in 7 days from the precipitating event.

\section{Discussion}

There are various definitions of abdominal compartment syndrome-a research definition which states that ACS is 
defined as a sustained intra-abdominal pressure $>20 \mathrm{mmHg}$ (with or without abdominal perfusion pressure $<60 \mathrm{mmHg}$ ) that is associated with new organ dysfunction [2]. In clinical practice, although desirable, it is not always possible to measure the intra-abdominal pressure [5].

For clinical purposes, intra-abdominal compartment syndrome is better defined as intra-abdominal hypertension (IAH) induced new organ dysfunction without a strict intraabdominal pressure threshold, since no intra-abdominal pressure can reliably diagnose all ACS [2].

Recognition of the acute IAH prior to the development of ACS is preferable as prompt treatment of the underlying cause can decrease the end-organ complications [6]. Development of abdominal compartment syndrome is the end of a pathophysiological spectrum, which is on a continuum of steady increases in intra-abdominal pressure [7]. At the severe end, it encompasses multiple biomediator generation which can result in multiorgan dysfunction syndrome.

In acute ACS, clinical symptoms can include any one of the following: cardiovascular, pulmonary, renal, hepatic, gastrointestinal, and central nervous system complications. Cardiovascular instability occurs due to the external pressure exerted on the vena cava significantly decreasing the venous return and cardiac output [8]. Respiratory system is affected due to a functional restriction of the diaphragmatic excursion. This results in significantly decreased respiratory compliance, hypoxemia, and $\mathrm{CO} 2$ retention.

Abdominal distension in this case affected both respiratory and cardiovascular systems. Although abdominal distension was not fully clinically recognized until the patient had failed extubation, diagnosis of ruptured bladder occurred after the respiratory failure and clinical ACS development.

The anaesthetic team noted raised airway pressure during anaesthesia. The standard approach of simultaneous diagnosis and management was taken but had a negative clinical yield. Increasing airway pressures are one of the known clinical signs of ACS [9]. This occurs due to extravasated intra-abdominal fluid having an effect on the respiratory function due to local compressive effect affecting functional residual capacity and causing significant bilateral lower lobe lung collapse.

There had been a failure to diagnose the abdominal expansion at this point. The patient had been covered with a warming blanket in a lithotomy position, and the anaesthetist did not conduct an abdominal examination.

A crude examination of the abdomen would have been helpful in this case.

It has been suggested that measurement of the abdominal girth and examination of the abdomen may be helpful when querying a bladder rupture. A more helpful approach in these cases may be to routinely examine the abdomen in patients having a rigid cystoscopy, particularly considering the high rate of asymptomatic bladder integrity compromise [10]. In a case illustrating acute ACS after a transurethral resection of the prostate, authors note the change of practice in urological procedures: they make a point of routinely assessing the abdomen visually with operating room lights on before extubation [10]. They also note newly implemented measurement of the input and output during the urological cases. Difficulty with ventilation can alert the anaesthetist to consider fluid extravasation in any endoscopic bladder procedure or arthroscopic hip procedure [10, 11].

In this case, bladder perforation was diagnosed due to the above respiratory presentation, failure to identify primary respiratory pathology, and, on a secondary survey, a rapidly expanding abdomen. Clinical suspicion was confirmed through the use of the FAST ultrasound scan, which demonstrated fluid in the abdomen and the subsequent imaging with the use of a CT scan. Clinical utility of ultrasound in this case was high. Anaesthesia as a specialty has recognized the importance of competent use of ultrasound in various diagnostic and management applications.

We have touched on some of the pathophysiological processes including local compressive effects on the cardiovascular and respiratory system as well as the biomediator generation resulting in acute intestinal distress and multiorgan failure [7]. Some of the main risk factors for development of ACS include damage control surgery in trauma patients. Others include intra-abdominal procedures, abdominal trauma, ruptured abdominal aneurysm, haemoperitoneum, liver transplant, and fluid extravasation after endoscopic procedures.

Management of ACS is broadly divided into supportive medical management strategies and more invasive surgical management [12]. Supportive medical management includes essential cardiovascular support as well as bowel lumen decompression, diuresis and improving of the abdominal wall compliance with sedation, and muscular paralysis.

There is still limited evidence as to whether treatment of IAH and even ACS in critically ill patients improves patient outcomes [7]. Well-designed studies comparing medical management of ACS with surgical decompression need to be performed as there is equipoise amongst clinicians as to the need for decompressive laparotomy in some conditions.

In this case, decision was made to not perform a postoperative laparotomy or a paracentesis. Either can be considered as definitive management of the surgical complication and free water release. This management decision was based on the successful results of supportive medical therapy including improving the abdominal wall compliance and diuresis. A supportive percutaneous tap was not considered by the management team at the time. This may have been beneficial due to decreasing the amount of fluid requiring diuresis and decreasing the physiological disturbance. In prior case reports of transurethral bladder perforation, all patients with extraperitoneal rupture were treated with an urgent laparotomy [3].

Intra-abdominal pressure in our patient was never measured; however with elevated airway pressures, respiratory failure due to localized compressive effect, and an eight-liter fluid diuresis, it is likely that this threshold was achieved. There have been alternative algorithms suggested [11]. In a recent case report, intra-abdominal extravasation occurred during hip joint laparoscopy. The authors suggested an algorithm use for patients suffering from intra-abdominal compartment syndrome during a hip joint arthroscopy. Following this algorithm, our patient could have been treated 
TABLE 1: Differences in resulting pathophysiology between transurethral bladder perforation and transurethral prostate perforation.

\begin{tabular}{|c|c|c|}
\hline & Transurethral bladder perforation & Transurethral prostate perforation \\
\hline Mode of fluid absorption & Absorbed across the peritoneal membrane & Direct intravascular entry \\
\hline IAH with potential ACS & Yes & No \\
\hline Fluid compartment affected & Extra- and intracellular, intravascular & Intravascular \\
\hline Respiratory & $\begin{array}{l}\text { Atelectasis due to abdominal girth expansion, } \\
\text { pulmonary oedema due to TBW overload }\end{array}$ & $\begin{array}{l}\text { Pulmonary oedema due to intravascular fluid } \\
\text { overload }\end{array}$ \\
\hline Cardiovascular & Relative hypovolaemia, hypotension & $\begin{array}{l}\text { Hypertension and bradycardia followed by } \\
\text { hypotension }\end{array}$ \\
\hline Neurological & Decreased GCS & Decreased GCS \\
\hline Gastrointestinal & Decreased perfusion due to ACS and hypotension & Unlikely to be affected \\
\hline
\end{tabular}

with abdominal paracentesis. Considering the similar pathophysiology of fluid extravasation, this may be an appropriate algorithm to follow in extraperitoneal bladder perforation and significant fluid accumulation.

There are previous short case series reports in the literature of intra-abdominal fluid accumulation following a bladder perforation [3]. All of the cases were recognized prior to completion of the surgery. All of the patients experiencing extraperitoneal perforation with severe symptoms were managed proactively with a surgical laparotomy. During a further literature review of cases, there have been reports of ACS following bladder neck rupture while performing a TURP [10]. In that situation, patient was managed with a laparotomy and had three liters of fluid extravasated in the abdomen in contrast to the more significant extravasation of eight to nine liters.

This patient suffered from a transurethral bladder syndrome, which results from the absorption of fluid down the osmotic gradient across the permeable peritoneal membrane. In contrast, in TURP syndrome, fluid absorption enters the intravascular circulation directly through compromised vessel integrity in a more immediate fashion.

TURP syndrome involves more rapid direct extravasation of fluid into the intravascular space. The full metabolic effects of intraperitoneal fluid absorption due to bladder perforation are therefore delayed. There has been a delineation between "TURBT" syndrome with the absorption of large volume of free water across the peritoneal membrane and "TURP" syndrome with its immediate consequences of intravascular overload.

Table 1 illustrates the differences in fluid pathophysiology with transurethral bladder perforation versus transurethral prostate perforation.

Sterile nonpyrogenic $1.5 \%$ glycine is preferred by urologists as it is nontoxic, nonhaemolytic with a refractive index close to that of water. When metabolised, glycine is transaminated to serine and deaminated to ammonia which is converted to urea [9]. Systemic absorption of large volumes of glycine results in the metabolic consequences of free water absorption and metabolism of ammonia to urea. This results in hyponatraemia due to a dilutional effect. There is also movement of sodium into the peritoneal fluid across the osmotic gradient, which results in greater hyponatraemia with potential for confusion and seizures $[13,14]$.
Free water overload distributes equally across the intravascular and extravascular components. Clinical expectations are those of TURP-like syndrome [3].

Lowest sodium was observed in the immediate postoperative course with the value recorded of $125 \mathrm{meq} / \mathrm{ml}$. This reflected a drop of 10 units, where the immediate preoperative sodium was $135 \mathrm{meq} / \mathrm{ml}$. The value took 7 days to normalize, with the return to $137 \mathrm{meq} / \mathrm{ml}$.

\section{Conclusion}

We have presented a case of primary acute abdominal compartment syndrome occurring intraoperatively, which was managed entirely through medical supportive treatment. There are suggestions that equipoise exists as to the need for surgical versus medical management in abdominal compartment syndrome, with a need for further valid studies.

As extraperitoneal fluid extravasation may occur during rigid cystoscopy, anaesthetists should consider monitoring clinically the abdominal girth routinely during these cases as well as use of ultrasound to assist their diagnosis.

It is important to differentiate clinically transurethral bladder perforation to transurethral prostate syndrome. Pathophysiology of the fluid entry and overload is different leading to alternative optimum medical management of either syndrome.

\section{Abbreviations \\ IAH: Intra-abdominal hypertension \\ ACS: Abdominal compartment syndrome \\ TURB: Transurethral bladder \\ TURP: Transurethral prostate \\ ETT: Endotracheal tube \\ TBW: Total body water.}

\section{Consent}

Written informed consent was obtained from the patient for publication of this case report and accompanying images.

\section{Conflicts of Interest}

The author declares that they have no conflicts of interest. 


\section{Acknowledgments}

The author would like to acknowledge the patient on whom this case report is based.

\section{References}

[1] M. D. Balbay, E. Çimentepe, A. Ünsal, Ö. Bayrak, A. Koç, and Z. Akbulut, "The actual incidence of bladder perforation following transurethral bladder surgery: editorial comment," Journal of Urology, vol. 174, no. 6, pp. 2260-2262, 2005.

[2] M. Gestring, Abdominal Compartment Syndrome, UpToDate, 2016, http://www.uptodate.com.

[3] Dorotta I., "Transurethral resection syndrome after bladder perforation," Anesthesia and Analgesia, vol. 97, pp. 1536-1538, 2003.

[4] D. Gaba, K. Fish, and S. Howard, Crisis Management in Anesthesiology, Churchill Livingstone; Second Edition, New York, 1994.

[5] M. Sugrue, "Abdominal compartment syndrome," Current Opinion in Critical Care, vol. 11, no. 4, pp. 333-338, 2005.

[6] K. Wong and C. F. Summerhays, "Abdominal compartment syndrome: a new indication for operative intervention in severe acute pancreatitis," International Journal of Clinical Practice, vol. 59, no. 12, pp. 1479-1481, 2005.

[7] D. J. Roberts, C. G. Ball, and A. W. Kirkpatrick, "Increased pressure within the abdominal compartment: intra-abdominal hypertension and the abdominal compartment syndrome," Current Opinion in Critical Care, vol. 22, no. 2, pp. 174-185, 2016.

[8] G. M. Luckianow, M. Ellis, D. Governale, and L. J. Kaplan, "Abdominal compartment syndrome: risk factors, diagnosis, and current therapy," Critical Care Research and Practice, vol. 2012, Article ID 908169, 8 pages, 2012.

[9] R. G. Hahn, "Fluid absorption in endoscopic surgery," British Journal of Anaesthesia, vol. 96, no. 1, pp. 8-20, 2006.

[10] M. M. Gaut and J. Ortiz, "Management of abdominal compartment syndrome after transurethral resection of the prostate," Brazilian Journal of Anesthesiology (English Edition), vol. 65, no. 6, pp. 519-521, 2015.

[11] K. Ciemniewska-Gorzela, T. Piontek, and A. Szulc, "Abdominal compartment syndrome: the prevention and treatment of possible lethal complications following hip arthroscopy: a case report," Journal of Medical Case Reports, vol. 8, no. 1, article no. $368,2014$.

[12] A. W. Kirkpatrick, D. J. Roberts, J. De Waele et al., "Intraabdominal hypertension and the abdominal compartment syndrome updated consensus definition and clinical practice guidelines from the world society of the abdominal compartment syndrome," Intensive Care Medicine, vol. 39, no. 7, pp. 11901206, 2013.

[13] J. Olsson and R. G. Hahn, "Simulated intraperitoneal absorption of irrigating fluid," Acta Obstetricia et Gynecologica Scandinavica, vol. 74, no. 9, pp. 707-713, 1995.

[14] R. G. Hahn, "Transurethral resection syndrome from extravascular absorption of irrigating fluid," Scandinavian Journal of Urology and Nephrology, vol. 27, no. 3, pp. 387-394, 1993. 


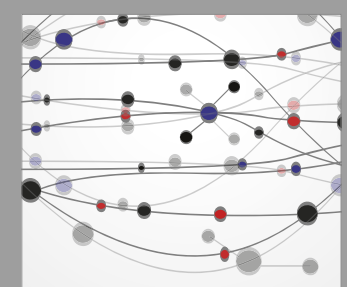

The Scientific World Journal
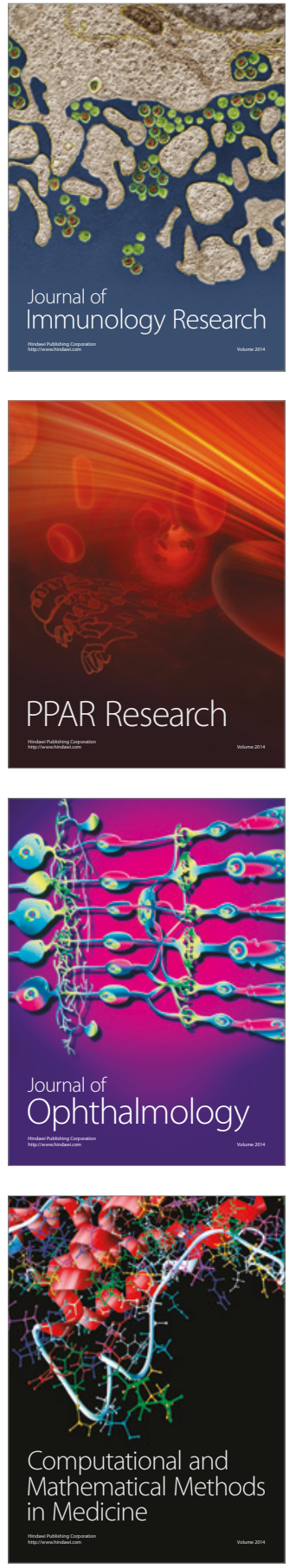

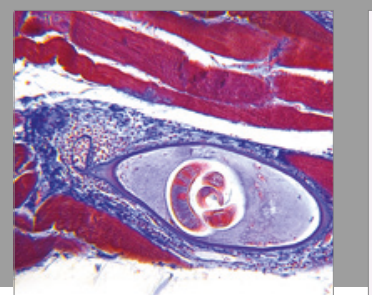

Gastroenterology Research and Practice
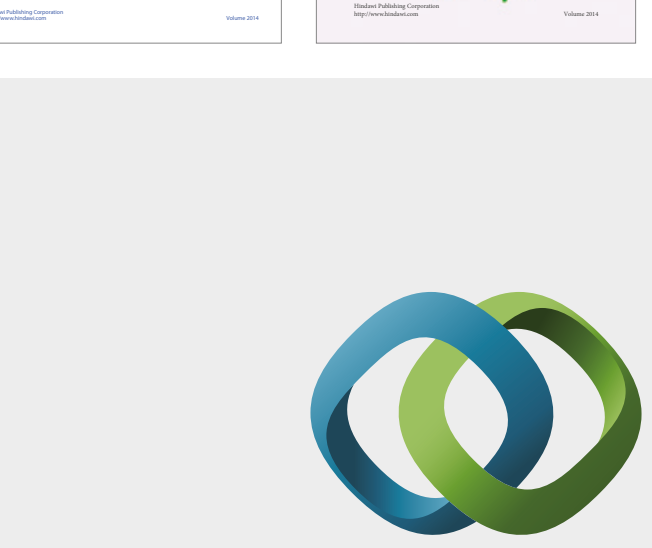

\section{Hindawi}

Submit your manuscripts at

https://www.hindawi.com
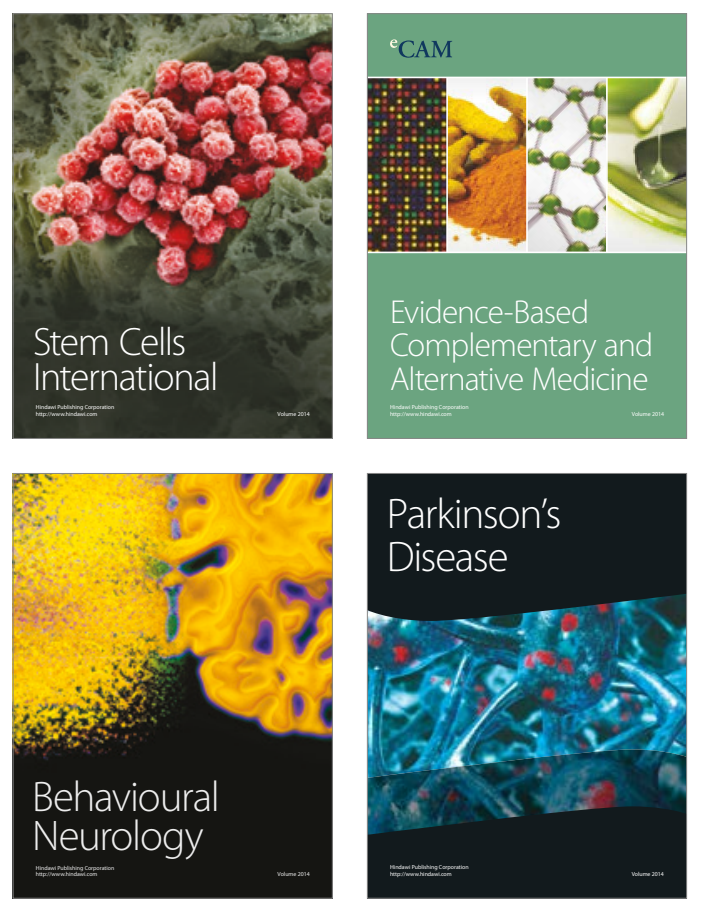
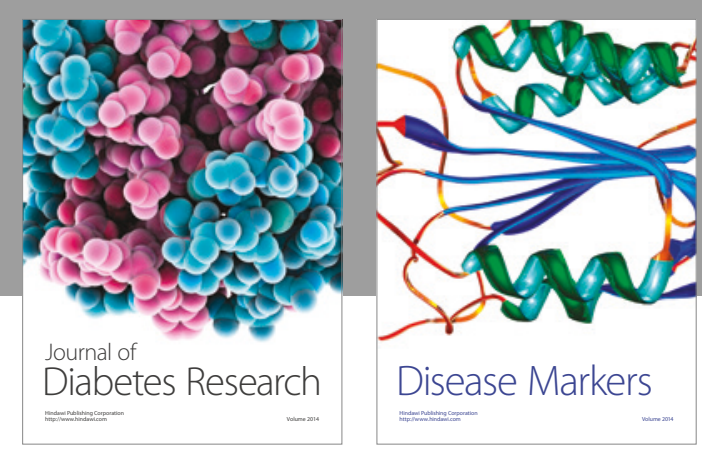

Disease Markers
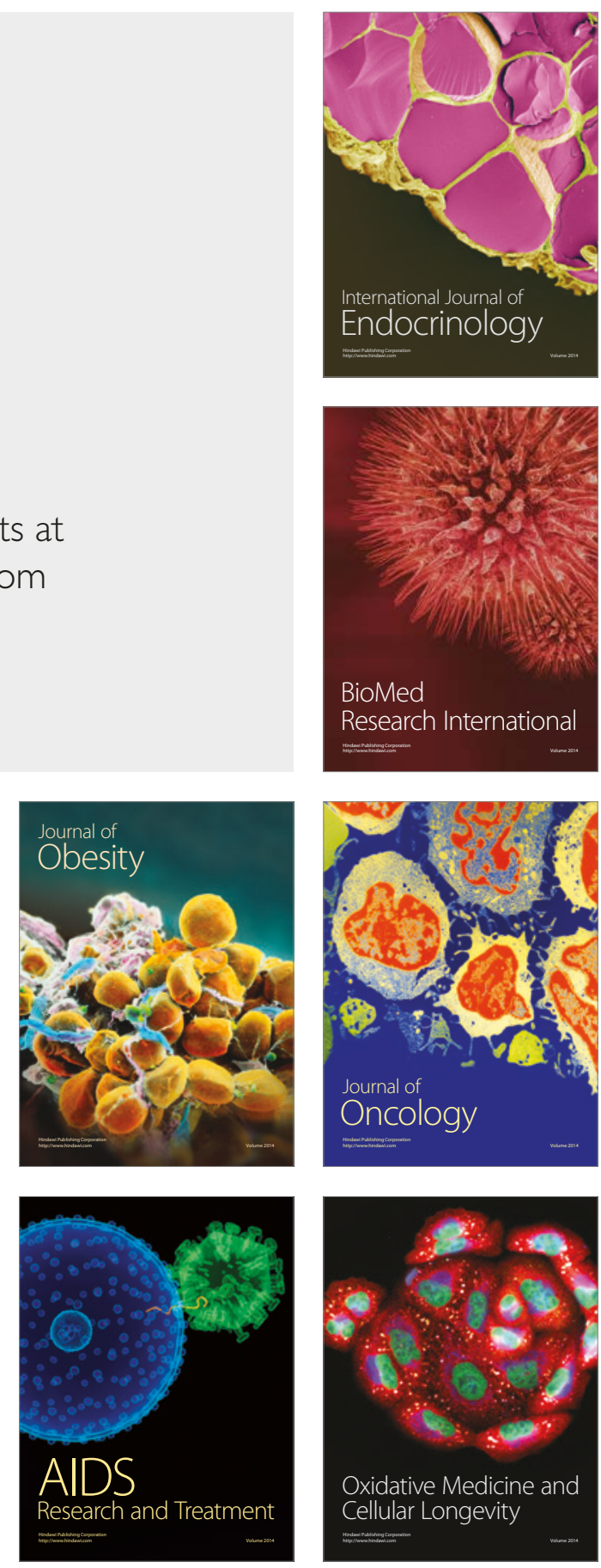